\title{
Production and Characterization of Biodegradable Povidone-iodine Microsphere as a Intramammary Disinfectant
}

\author{
Hee-Myung PARK ${ }^{1)}$ and Hong-Ryul HAN ${ }^{2)}$ \\ ${ }^{1)}$ Department of Veterinary Internal Medicine, College of Veterinary Medicine, Konkuk University, 1 Hwayang-dong, Gwanggin-gu, \\ Seoul, Korea 143-701 and ${ }^{2}$ Department of Veterinary Internal Medicine, College of Veterinary Medicine, Seoul National University, \\ Sinlimdong San 56-1, Kwanak-Gu, Seoul, Korea 151-080
}

(Received 8 August 2001/Accepted 8 April 2002)

\begin{abstract}
Microspheres composed of biocompatible, biodegradable poly dl-lactide-co-glycolide (DL-PLGA) and Povidone-iodine were evaluated as an intramammary disinfectant delivery system in vitro prior to infusion into mammary glands. Microsphere was prepared by solvent evaporation method and particle size, morphology and in vitro release kinetics were examined. The microspheres were ranged in size from $25 \mu \mathrm{m}$ to $155 \mu \mathrm{m}$ (mean diameter $=65.7 \mu \mathrm{m}$ ). Povidone-iodine was dispersed on the surface of microsphere and microsphere was spherical in shape with a smooth surface. The yield of microsphere was $57.3 \%$ and the encapsulation efficiency was $69.6 \%$. In in vitro release study, a burst effect $(50.9 \%)$ was observed during the first two days and a sustained release then continued for the next 28 days. Results of the present study demonstrated that microsphere have the potential for new intramammary disinfectant formulations that can provide increased efficacy of therapy against mastitis pathogens.
\end{abstract}

KEY WORDS: DL-PLGA, intramammary disinfectant, mastitis, Povidone-iodine.

J. Vet. Med. Sci. 64(8): 739-741, 2002

Despite worldwide efforts, bovine mastitis has remained the most economically important disease of dairy cattle [7, 10, 11]. The implementation of effective measures for prevention and control of the disease has markedly reduced subclinical and clinical mastitis [9]. However, there is still a great need for effective therapeutic measures against mastitis, although various preventive measures and management practices are effective. During the lactating period, dairy cows are infected with a variety of mastitis pathogens including environmental and contagious bacteria.

Removal of pathogenic bacteria from udders of lactating cows is lower compared to cure rate of dry cow therapy. In addition, antibiotic residue in milk occurs during the therapy of lactating cows and subsequently, affected milk should be discarded. Moreover, after systemic antibiotic treatment, antibiotics in milk is not likely to exist with high concentration in udders of lactating cows due to continuous milking of cows. Thus, once lactating cows are infected with mastitis, affected cows are dried off early or are initiated with antibiotic treatment. Therefore, many mastitis researches have been focused on the development of more efficient therapy and management for dry cows. Povidone-iodine used in this study has several advantages of antibacterial and antifungal effects. In addition, this has been safely and broadly used as a teat-dipping disinfectant in dairy farms of our country.

Therefore, in this study, we examined the potential exploitation of biodegradable, biocompatible, and sustained release Povidone-iodine-loaded microsphere as a intramammary disinfectant for dry cow therapy prior to intramammary infusion .

Microspheres were prepared by solvent evaporation method described previously [1]. Briefly, $5 \mathrm{ml}$ of Povidoneiodine (10\%, w/v) (Sungkwang, Suweon, Kyunggi, Korea) and $2.5 \mathrm{~g}$ of DL-PLGA (65:35) (Sigma, St. Louis, MO,
U.S.A.) in $50 \mathrm{~m} l$ of dichlormethane (Aldrich Chemicals Co., Milwaukee, WI, U.S.A.) were mixed and emulsified at $10,000 \times \mathrm{g}$ (IKA Labortechnik, Hanover, Germany) for 5 min to form a first emulsion (w/o). This first emulsion was then added to $1 \mathrm{~L}$ of aqueous polyvinyl alcohol solution $(1 \%, w / v)$ (PVA, Sigma) and emulsified according to the same method of first emulsion step described above. Second emulsion was poured in $4 \mathrm{~L}$ of distilled water (DW) and stirred at $200 \mathrm{rpm}$ for $8 \mathrm{hr}$ under room temperature to allow the solvent evaporation and microsphere formation. Microspheres were washed three times with DW by centrifugation at $1,000 \times \mathrm{g}$ for $10 \mathrm{~min}$ and then the washed microsphere was lyophilized.

To determine the particle size, the microsphere suspension was diluted with PBS at a ratio of 1:100 and determined using a Coulter counter (Multisizer, Coultronics, Margency, France) according to the previous method [1]. Morphological analysis and surface appearance of microspheres were examined as described previously [3, 4, 12]. Briefly, samples for scanning electron microscope (SEM) (Jeol, Paris, France) were mounted on metal stubs and coated with gold to a thickness of 20-50 nm. Pictures were taken and the diameter of microsphere was determined according to a reference scale. Suspended samples in phosphate-buffered saline (PBS, pH 7.4) for optical microcopy (Olympus, Paris, France) were mounted on convexed slide glass and PBS was aspirated with $5 \mathrm{ml}$-syringe. And the microsphere was then examined.

The yield of microsphere was determined by comparing the total weight of lyophilized microsphere with the combined weight of co-polymer and Povidone-iodine. The amount of Povidone-iodine entrapped in microspheres was determined using a procedure described previously [2]. Briefly, $5 \mathrm{mg}$ of Povidone-iodine-loaded microspheres was 
suspended in $20 \mu l$ of $0.1 \mathrm{~N} \mathrm{NaOH} / 1 \%$ sodium dodecyl sulfate (SDS) and boiled at $100^{\circ} \mathrm{C}$ for $15 \mathrm{~min}$. The samples were slowly cooled at room temperature and centrifuged at $15,000 \times \mathrm{g}$ for $10 \mathrm{~min}$. The released amount of Povidoneiodine in the supernatant was determined by the spectrophotometer (Hitachi, Tokyo, Japan) at $260 \mathrm{~nm}$. And the encapsulation efficiency was then determined by the percentage of encapsulated Povidone-iodine in total Povidone-iodine.

This in vitro release study was performed according to the method described previously [2, 12]. Briefly, $30 \mathrm{mg}$ of microspheres were placed in $5 \mathrm{~m} l$ of plastic tube. Five $\mathrm{m} l$ of PBS (pH 7.4) were added and agitated with $100 \times \mathrm{g}$ at $37^{\circ} \mathrm{C}$. Samples were collected periodically at day $0,1,2,4$ and 7 days respectively. After that, samples were collected once a week for 3 weeks. The colllected samples were centrifuged at $6,000 \times \mathrm{g}$ for $20 \mathrm{~min}$ and the supernatant was obtained. The supernatant was analysed spectrophotometrically at 260 $\mathrm{nm}$ (Hitachi). The in vitro release study was at least three times performed independently in triplicate and the released percentage was determined by the theoretical concentration.

The diameter of microsphere was determined using Coulter counter. The microspheres were ranged in size from $25 \mu \mathrm{m}$ to $155 \mu \mathrm{m}$ (Fig. 1). They could be divided into three size groups: $<50 \mu \mathrm{m}(35 \%), 50-100 \mu \mathrm{m}(50 \%)$, and $>100$ $\mu \mathrm{m}(15 \%)$. Mean diameter and coefficient of variation were $65.7 \mu \mathrm{m}$ and 0.54 , respectively. Microspheres prepared in this study were also examined by SEM. The results indicated that microsphere was spherical in shape with a smooth surface (Fig. 2). In addition, microsphere showed no porous structures on the surface (Fig. 2).

The yield of microsphere was $57.3 \%$ (final product weight $1.72 \mathrm{~g}$ : total weight $3.0 \mathrm{~g}$ ) by solvent evaporation technique in this study. The encapsulation efficiency of Povidone-iodine in microspheres was $69.6 \%$ (released Povidone-iodine concentration, $3.48 \mathrm{mg}$ : total microsphere weight, $5 \mathrm{mg}$ ). Results of the in vitro release study of Povidone-iodine-loaded microspheres are shown in Fig. 2. A burst effect (50.9\%) was observed during the first two days. A sustained release then continued for the next 28 days, suggesting that the degradation of microspheres is controlled by DL-PLGA matrix.

Several aspects of microsphere consisted of DL-PLGA make us attractive as a novel drug delivery system for therapeutics. One important aspect is that DL-PLGA is in the class of biodegradable and biocompatible copolymers from which resorbable suture material are made [8]. The other is that there is no severe tissue reaction, suggesting that this microsphere can be absorbed into tissue with a sustained release action [8]. Thus many studies have focused on the development of encapsulation skill for therapeutics and vaccinology [3]. In this study, we examined the possibility that microsphere of DL-PLGA could be used as an intramammary disinfectant for dry period in mastitis.

Results from analysis of microsphere morphology demonstrated that microsphere was spherical in shape and had smooth surface. This latter observation is inconsistent with the reports that encapsulated microspheres were highly

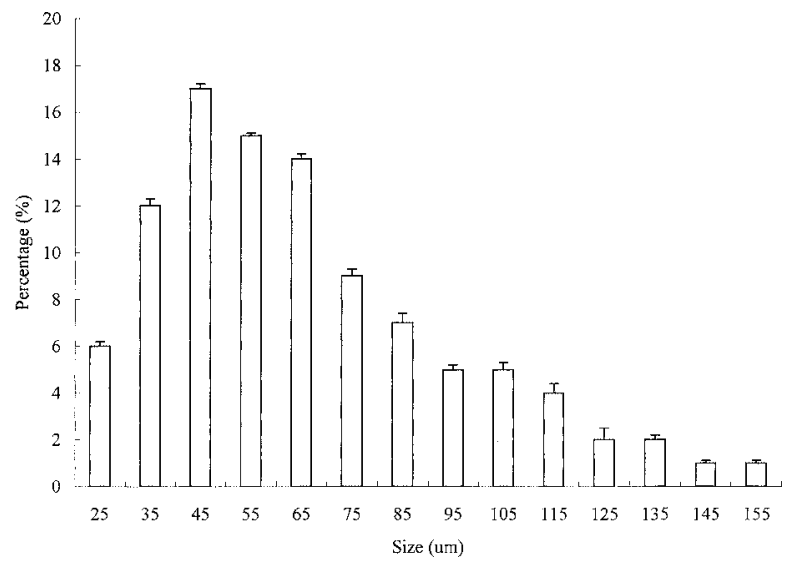

Fig. 1. Particle size distribution of d, 1-poly(lactide-co-glycolide) (DL-PLGA) microspheres. The diameter of microsphere was determined using Coulter counter. Error bar means standard deviation.

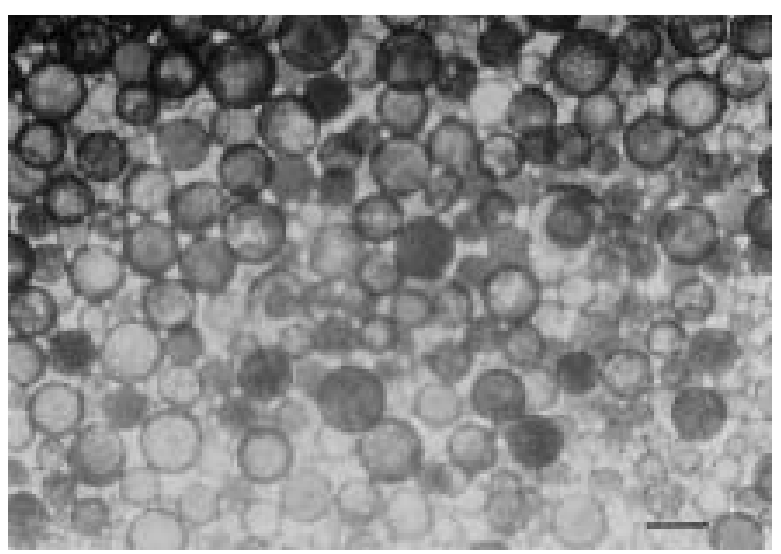

Fig. 2. Optical microscopy of Povidone-iodine-loaded DLPLGA microsphere. The morphology of microspheres shows no porous structure. The size bar represents $100 \mu \mathrm{m}$.

porous [5]. This could be explained by the facts that according to the ratio of lactide and glycolide, surface characteristics of microsphere varied [5].

Results of the present study exhibited that encapsulation and yield of microsphere was $69.6 \%$ and $57.3 \%$, respectively. This is inconsistent with a previous report [6] that showed that for the spray-dried the encapsulation efficiency was $75 \%$. This difference could be explained by the facts that encapsulation efficiency was mainly influenced by the polymer ratio (lactide:glycolide), concentration of PVA and encapsulation methods [6].

In vitro release kinetics of Povidone-iodine-loaded microsphere showed a burst effect (50.9\%) during the first two days and a sustained release for the next 28 days in this study. However, an earlier study [5] reported that BSAloaded microsphere showed approximately $30 \%$ of burst effect during the first two days. This difference of burst time could be due to the different surface characteristics and 
size of microspheres. BSA-loaded microspheres released BSA through the highly porous structure slowly while the release of Povidone-iodine-loaded microsphere may be mainly controlled by diffusion during the initial period. This assumption is supported by the facts that, on the optical microscope, Povidone-iodine was dispersed on the surface of microspheres. Thus, the reason that Povidone-iodineloaded microsphere showed high bursting effects compared to that of BSA-loaded microsphere could be explained by the facts that Povidone-iodine-dispersed structure contributed a rapid diffusion through the surface of microsphere at the initial stage. However, the continued release for 28 days might be controlled by DL-PLG degradation at the later stage.

Results of the present study demonstrated that, because this microsphere exhibited a sustained and orderly release in vitro, sustained release of Povidone-iodine from a biodegradable microsphere could be used for dry cow therapy if it is possible to extend the release time of microsphere to 2 months.

In conclusion, this novel technique showed the possibility that the amount of infused antibiotics and vaccine antigen could be reduced whereas the time of action could be increased in udder of dry cows. However, the encapsulation method of microsphere for vaccine antigen and antibiotics should be further studied by altering several parameters.

\section{REFERENCES}

1. Alonso, M., Cohen, J., Park, S., Gupta, T. G., Siber, R. K. and Langer, G. R. 1993. Pharmacol. Res. 10: 945-953.

2. Blanco-Prieto, M., Fattal, J., Gulik, E., Dedieu, A., Roques, J. C. and Couvreur, B. P. 1997. J. Controlled Release. 43: 81-87.

3. Cleland, J. L. 1998. Biotechnol. Prog. 14: 102-107.

4. Han, H. R., Youn, S. K. and Park, H. M. 1999. Korean J. Buiatrics. 4: 25-29.

5. Ho, T. Y., Wu, S. L., Hsiang, C. H., Hou, B. H. and Hsiang, C. Y. 1998. J. Chin. Soc. Vet. Sci. 24: 128-134.

6. Johansen, P., Moon, L., Tamber, H., Merkle, H. P., Gander, B. and Sesardic, D. 2000. Vaccine 18: 209-215.

7. Miller, G. Y. and Dorn, C. R. 1990. Prev. Vet. Med. 8: 171178.

8. Redding, T. W., Schally, A. V., Tice, T. R. and Myers, W. E. 1984. Proc. Natl. Acad. Sci. U.S.A. 81: 5845-5851.

9. Shpigel, N. Y., Levin, D., Winkler, M., Saran, A., Ziv, G. and Bottner, A. 1997. J. Dairy Sci. 80: 318-323.

10. Weigler, B. J., Hird, D. W. and Sischo, W. M. 1990. J. Am. Vet. Med. Assoc. 196: 1945-1949.

11. Wilesmith, J. W., Francis, P. G. and Wilson, C. D. 1986. Vet. Rec. 118: 119-125.

12. Witschi, C. and Doelker, E. 1998. J. Controlled Release 51: 327-341. 